\title{
A cross-species assay demonstrates that reward responsiveness is enduringly impacted by adverse, unpredictable early-life experiences
}

\author{
Brian D. Kangas (iD ${ }^{1,2,7}$, Annabel K. Short ${ }^{3,4,7}$, Oanh T. Luc ${ }^{2}$, Hal S. Stern ${ }^{5}$, Tallie Z. Baram (D) ${ }^{3,4}$ and Diego A. Pizzagalli (D) ${ }^{1,6 \bowtie}$ \\ (c) The Author(s), under exclusive licence to American College of Neuropsychopharmacology 2021
}

Exposure to early-life adversity (ELA) is associated with several neuropsychiatric conditions, including major depressive disorder, yet causality is difficult to establish in humans. Recent work in rodents has implicated impaired reward circuit signaling in anhedoniclike behavior after ELA exposure. Anhedonia, the lack of reactivity to previously rewarding stimuli, is a transdiagnostic construct common to mental illnesses associated with ELA. Here, we employed an assay of reward responsiveness validated across species, the Probabilistic Reward Task (PRT). In the PRT, healthy participants reliably develop a response bias toward the more richly rewarded stimulus, whereas participants with anhedonia exhibit a blunted response bias that correlates with current and future anhedonia. In a well-established model of ELA that generates a stressful, chaotic, and unpredictable early-life environment, ELA led to blunted response biases in the PRT in two separate cohorts, recapitulating findings in humans with anhedonia. The same ELA rats had blunted sucrose preference, further supporting their anhedonic-like phenotypes. Probing the aspects of ELA that might provoke these deficits, we quantified the unpredictability of dam/pup interactions using entropy measures and found that the unpredictability of maternal care was significantly higher in the ELA groups in which PRT and sucrose preference reward deficits were present later in life. Taken together, these data position the PRT, established in clinical patient populations, as a potent instrument to assess the impact of ELA on the reward circuit across species. These findings also implicate the unpredictability of maternal signals during early life as an important driver of reward sensitivity deficits.

Neuropsychopharmacology (2022) 47:767-775; https://doi.org/10.1038/s41386-021-01250-9

\section{INTRODUCTION}

Sensitivity to reward is modulated by prior experiences, particularly during early developmental periods [1-11]. In the United States, over $30 \%$ of children experience some type of early-life adversity (ELA) related to poverty, trauma, and chaotic environments [12] and these events have been associated with poor cognitive and emotional health [13-16]. Anhedonia, the loss of pleasure or lack of reactivity to previously rewarding stimuli, is another consequence associated with ELA. Adversity during childhood has been shown to alter (in rodents) or predict (in humans) the development of the reward system and impair reward responsiveness later in life [17-23]. Important questions remain, however, regarding causality in human studies, and the mechanisms and salient signals of adversity in both humans and animal models.

Blunted reactivity to previously rewarding activities is also commonly associated with major depressive disorder [24], bipolar disorder [25], schizophrenia [26], post-traumatic stress disorder $[27,28]$, and substance use disorders [29]. Given the prevalence of anhedonia across diverse neuropsychiatric conditions and the absence of effective pharmacotherapeutic treatment options, an active research domain has emerged dedicated to examining the features and mechanisms of this highly heterogeneous construct [30]. For decades, clinical assessments of anhedonia have relied heavily on self-report questionnaires such as the Snaith-Hamilton Pleasure Scale [31], Chapman Physical and Social Anhedonia Scales [32], and Fawcett-Clark Pleasure Capacity Scale [33]. In rodents, assays such as the sucrose preference task and intracranial self-stimulation have been commonly employed to assay anhedonic-like phenotypes [34]. However, they depart significantly from diagnostic instruments used in the clinic. Therefore, recent work has focused on the development and empirical validation of coordinated laboratory assays in clinical patients and laboratory animals designed to quantify more objectively responsiveness to reinforcement contingencies as a means to identify deficits. Here, the expectation is that bidirectional alignment in methodologies across species will advance our understanding of the mechanisms involved in disorders in which anhedonia is prominent and accelerate the discovery of treatment options.

\footnotetext{
${ }^{1}$ Department of Psychiatry, Harvard Medical School, Boston, MA, USA. ${ }^{2}$ Behavioral Biology Program, McLean Hospital, Belmont, MA, USA. ${ }^{3}$ Department of Anatomy and Neurobiology, University of California-Irvine, Irvine, CA, USA. ${ }^{4}$ Department of Pediatrics, University of California-Irvine, Irvine, CA, USA. ${ }^{5}$ Department of Statistics, University of California-Irvine, Irvine, CA, USA. ${ }^{6}$ Center for Depression, Anxiety, and Stress Research, McLean Hospital, Belmont, MA, USA. ${ }^{7}$ These authors contributed equally: Brian D. Kangas, Annabel K. Short. ${ }^{\circledR}$ email: dap@mclean.harvard.edu
} 
P2-P9 Control/ELA
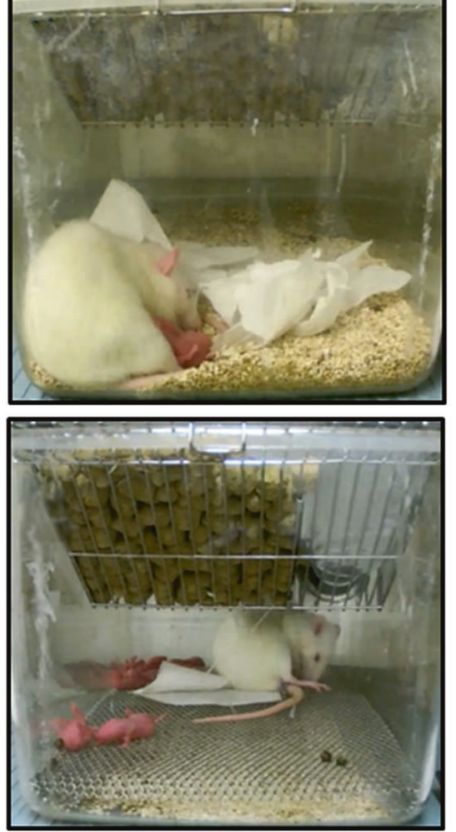

P75-90 Sucrose Preference 1

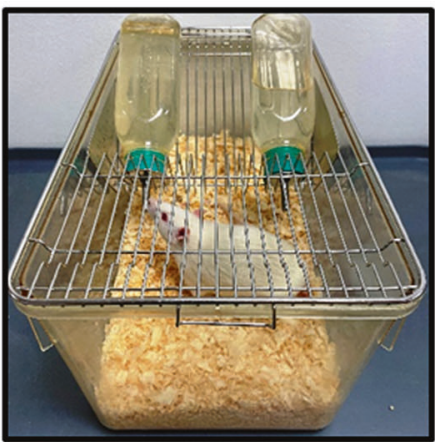

$\sim$ P200

PRT

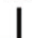

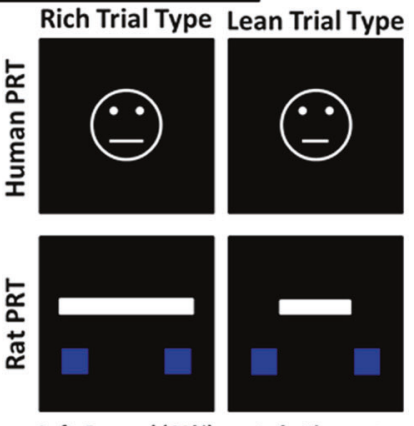

Left: Reward (60\%) Left: Timeout Right: Timeout Right: Reward (20\%)

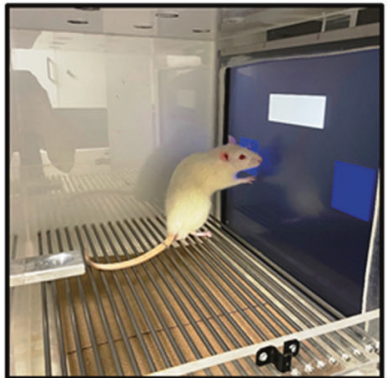

Fig. 1 Study overview. Experimental timeline across postnatal $(P)$ days and task schematics for the ELA limited bedding and nesting paradigm, sucrose preference test, and touchscreen-based PRT.

One such approach that has been chosen as a recommended task for the reward learning subdomain in the latest revision [35] of the RDoC matrix [36] is the Probabilistic Reward Task (PRT) [37, modified after 38]. In this computerized task, human participants make rapid discriminations between two lines that differ in length, represented as the mouth on a cartoon face (see Fig. 1). Unbeknownst to the participants, asymmetric 3:1 probabilistic reinforcement contingencies are programmed such that correct responses to one line length are rewarded $60 \%$ of the time, whereas correct responses to the other line length are rewarded only $20 \%$ of the time. As predicted by signal detection theory [39-41], healthy participants reliably develop a response bias toward the more richly rewarded stimulus; however, participants with anhedonia consistently exhibit a blunted response bias that has been found to correlate with current and predict future anhedonia in depression and other neuropsychiatric conditions [42-46].

The ability of the PRT to objectively quantify reward responsiveness in clinical populations with anhedonia has inspired reverse translation of this task for rodents $[47,48]$ and nonhuman primates [49]. Studies in laboratory animals have already elucidated functions related to neurochemical signaling systems [50], revealed expected reductions in reward responsiveness following social defeat stress [51], and documented increases in sensitivity following treatment with drugs known to enhance hedonic tone $[48,49]$. In order to examine the relationship between ELA and anhedonic phenotypes, the present studies employed the touchscreen-based PRT in rats to evaluate whether the limited bedding and nesting protocol (a model of chronic ELA as a result of unpredictable maternal care) would be associated with a blunting in reward responsiveness that persists into adulthood. PRT outcomes were also compared to traditional sucrose preference measures and entropy metrics of dam/pup interactions as a means to determine specificity in blunted reward responsiveness with predictability in maternal interaction.

\section{METHODS}

Subjects

A total of 32 male Spraque Dawley rats across two cohorts and born from six timed-pregnant dams (delivered embryonic day 15; Envigo, Livermore, CA) served in the present studies. Cohort 1 was comprised of 6 control and 6 ELA rats and Cohort 2 was comprised of 11 control and 9 ELA rats. The experimental timeline and task schematics are summarized in Fig. 1. Following standard or ELA rearing protocols, entropy measurements, and subsequent sucrose preference testing at the University of California-Irvine detailed below, Cohort 1 was sent via 1-day shipping from California to Massachusetts in December 2019 and Cohort 2 was sent using the same carrier in December 2020. Upon arrival at McLean Hospital, Cohort 1 was quarantined for 60 days in an isolated climate-controlled vivarium bay with unrestricted access to rodent chow and water. Cohort 2 was quarantined under identical conditions but, due to COVID-19-related delays in sentinel testing, was not released for approximately 90 days. Following clearance from quarantine, subjects were transferred to a larger vivarium with otherwise identical housing conditions. Subjects continued to have unrestricted access to water in their home cage; however, to establish sweetened condensed milk as a reinforcer, subjects were food-restricted via daily post-session portions of $10-15 \mathrm{~g}$ of rodent chow. All vivarium technicians and research assistants responsible for animal husbandry duties and conducting the behavioral studies were blinded to the subjects' group assignment (i.e., control or ELA). See Table 1 for additional birth date and developmental weight data across these studies. The protocol was approved by the Institutional Animal Care and Use Committee at McLean Hospital and in accordance with guidelines from the Committee on Care and Use of Laboratory Animals of the Institute of Laboratory Animals Resources, Commission on Life Sciences (2011) [52].

\section{Early-life adversity paradigm}

Six dams across two cohorts (two in Cohort 1, four in Cohort 2) were randomly assigned as either control or ELA on P2. Pups were cross-fostered between litters born within $12 \mathrm{~h}$ to create litters of 12 with equal numbers of sexes where possible. ELA was imposed using the limited bedding and nesting paradigm, which consists of limiting nesting and bedding materials in cages between P2 and P9 as described previously [53-55]. For the ELA group, a plastic-coated mesh platform was placed $2.5 \mathrm{~cm}$ above the floor of a standard cage. Cob bedding was reduced to cover the 


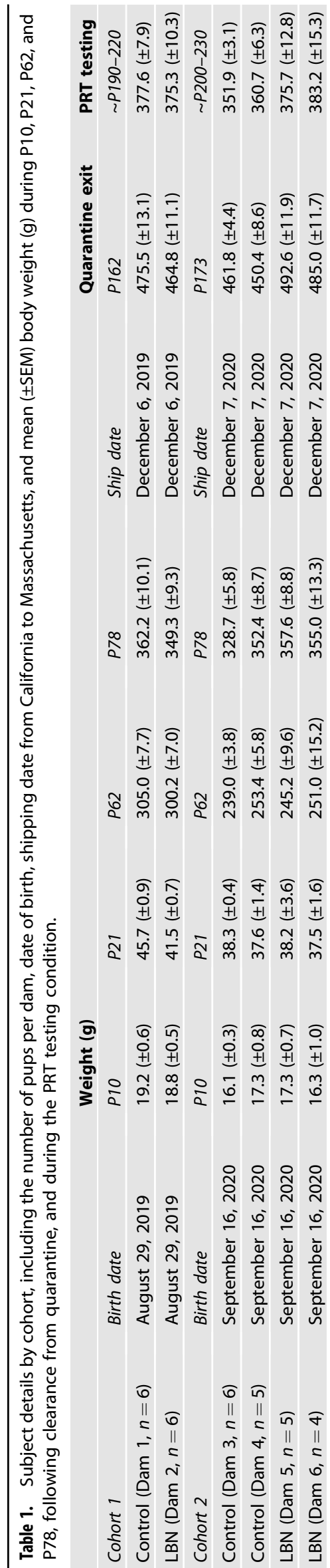

cage floor sparsely, and one-half of a single paper towel was provided for nesting material on the platform. Control dams and litters resided in standard cages containing ample cob bedding and one whole paper towel which dams shred to create a nest. See Fig. 1 for a representative photograph of control and ELA rearing conditions. Both control and ELA cages were undisturbed during P2-P9, housed in temperature- and humidity-controlled rooms. On P10, all groups were transferred to standard cages. Rats were weaned on P21 and then group housed. Male rats were used in these experiments and the females were the subjects of other studies aimed at understanding female-specific effects of ELA [7].

\section{Characterization of maternal unpredictability}

Maternal behaviors were assessed via home cage observation for 60 -min periods during P3-P6. Mothers were identified as performing one of seven different behaviors: licking/grooming pups, carrying pups, eating, nursing (in high- or low-arched posture), nest building, off pups, or self-grooming. Behaviors were recorded if lasting for a minimum of $3 \mathrm{~s}$. Coding of maternal behaviors was performed live and from video recordings by two experimenters blinded to the group assignment. Recorded videos were manually coded using Behavioral Observation Research Interactive Software [56]. Entropy is a natural summary measure of randomness or unpredictability of a probability distribution and the entropy rate can be used to measure the unpredictability of a sequence of observed behaviors. To calculate the maternal entropy for each dam, the dam's sequence of behaviors was characterized using the empirical transition matrix $\left\langle p_{i j}\right\rangle_{i j}=$ $1 \ldots 7$ of conditional probabilities of moving from one behavior (behavior $i$ ) to another (behavior $j$ ). The entropy rate of each dam was calculated from the transition matrix as previously described $[8,57]$ and detailed below.

\section{Sucrose preference testing}

When animals were two months of age, a preference for a weak sucrose solution ( $1.5 \%$ in filtered water) in comparison to water was performed in a limited two-bottle choice setting as described previously [3, 8]. Rats were acclimated to individual housing for 7 days with ad libitum food and given free access to two bottles containing $50 \mathrm{ml}$ of filtered water and were handled daily for approximately $2 \mathrm{~min}$. On day 8 , rats were provided with two bottles, one containing $50 \mathrm{ml}$ of filtered water and the other $50 \mathrm{ml}$ of $1.5 \%$ sucrose solution during a 1-week period. The left/right position of the bottles was interchanged daily to avoid effects from a side-bias. Fluid (water and sucrose) consumption was recorded each morning, correcting for evaporation/spillage. Following completion of the task, rats were returned to their original housing groups.

\section{PRT training and testing}

Following arrival at McLean Hospital, subsequent clearance from quarantine, and implementation of food restriction conditions, PRT training and testing protocols were initiated. Empirical validation and task optimization of the touchscreen-based rat PRT can be found in [48]. Details of the rat touch-sensitive experimental chamber can be found in [58] and a task schematic and photograph are presented in Fig. 1.

Line length discrimination training. Discrete trials began with the presentation of a white line on a black background, with its lower edge presented $3 \mathrm{~cm}$ above the $5 \times 5 \mathrm{~cm}$ left and right blue response boxes. The length of the line was either $600 \times 120 \mathrm{px}(31.5 \times 6.5 \mathrm{~cm}$ : long line $)$ or $200 \times 60 \mathrm{px}(10.5 \times 3.25 \mathrm{~cm}$ : short line). Long and short line length trial types varied in a quasi-random manner across 100-trial sessions such that there were exactly 50 trials of each type, but a given trial type would not be presented more than five times in a row. Subjects were trained to respond to the left or right response box depending on the length of the white line (long line: respond left, short line: respond right, or vice versa). Response box designation was counterbalanced across subjects. During line length discrimination training, each correct response was reinforced with $0.1 \mathrm{ml}$ of $30 \%$ sweetened condensed milk, paired with an $880-\mathrm{ms}$ yellow screen flash and a $440 \mathrm{~Hz}$ tone, and followed by a 5-s blackout period, whereas each incorrect response immediately resulted in a 10-s blackout period. A correction procedure was implemented during initial discrimination training in which each incorrect trial was repeated until a correct response was made [59], and was discontinued after $<10$ repeats of each trial type occurred in two consecutive sessions. Discrimination training sessions continued without correction until accuracies for both line length trial types were $\geq 80 \%$ correct for two consecutive sessions, concordant with the performance criteria in previous human PRT studies $[37,43,44]$. After this criterion was met, PRT testing commenced. 

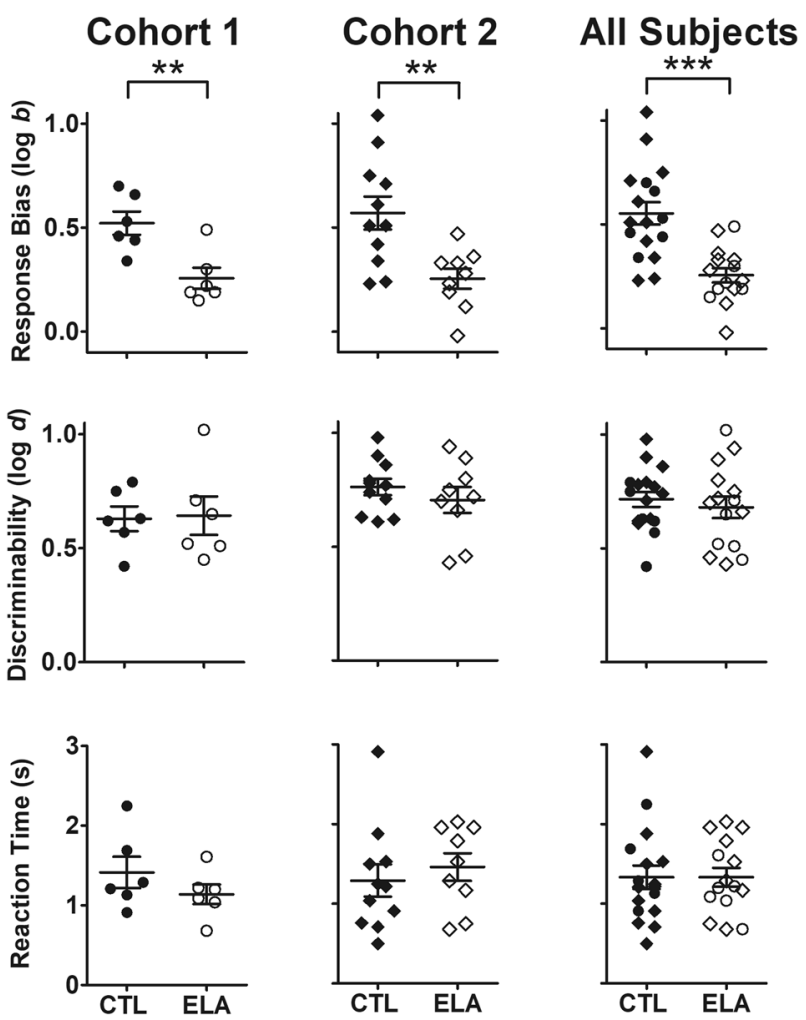

Fig. 2 PRT outcomes by group and cohort. Effects of control (filled symbols) and ELA (unfilled symbols) rearing conditions in Cohort 1 (left panels; circles; control, $n=6, \mathrm{ELA}, n=6$ ), Cohort 2 (middle panels; diamonds; control, $n=11$, ELA, $n=9$ ), and both cohorts combined (right panels; control, $n=17$, ELA, $n=15$ ) on response bias (upper row), task discriminability (middle row), and reaction time (lower row). Horizontal lines represent group mean $( \pm S E M)$ and data points represent values for individual subjects. ${ }^{* *} p<0.01$, ${ }^{* * *} p<0.0001$.

PRT testing. Subjects were exposed to a 5-session protocol using 3:1 probabilistic reinforcement contingencies such that a correct response to one of the line lengths (long or short) was reinforced $60 \%$ of the time (rich stimulus), whereas a correct response to the other line length was reinforced $20 \%$ of the time (lean stimulus). Incorrect responses were never reinforced. The line length associated with the rich and lean contingency was determined for each subject during their final two line length discrimination training sessions by examining their accuracies and designating the line length with a higher mean accuracy as the stimulus to be rewarded on the lean schedule. This approach was expressly designed to examine the effects of ELA on response bias generated by responsivity to asymmetrical probabilistic contingencies, rather than the amplification of a preexisting inherent bias that is a function of uncontrolled variables.

\section{Data analysis}

Maternal entropy. Entropy was calculated using $\mathrm{R}$ (version 4.0.3; code available at https://github.com/bvegetabile/entropyrate). Specifically, the entropy rate of each dam was derived from the transition matrix $[8,57]$ as follows:

$E=-\sum_{i=1}^{M} \pi_{i} \sum_{j=1}^{M} p_{i j} \log \left(p_{i j}\right)$

where $p_{i j}$ is the conditional probability that maternal behavior $j$ is observed next after a mother is observed performing behavior $i, \pi_{i}$ is the frequency with which behavior $i$ is observed, and $M(=7)$ is the number of different behaviors. Entropy across P3-P6 was analyzed using two-way repeatedmeasures ANOVA, with time as a repeated factor. Average entropy was taken across the 4 days and tested for significance across the two conditions using Student's $t$-test $(p<0.05)$ for differences between means.

Sucrose preference. Preference for the sucrose solution was calculated as (sucrose consumption [ml]/total fluid consumption $[\mathrm{ml}]) \times 100$ and averaged across the 6 days. Due to missing values, preference over time was analyzed using a mixed-effects model (Restricted Maximum Likelihood), with time as a repeated factor. Average preference over 6 days was tested for significance using Student's t-test $(p<0.05)$ for differences between means.

PRT. The implementation of probabilistic contingencies yields two primary dependent measures: response bias and discriminability, which can be quantified using equations derived from signal detection theory [39-41] by examining the number of correct and Incorrect responses for Rich and Lean trial types. Response bias is calculated using the following $\log b$ equation:

$\log b=0.5 * \log \left(\frac{\left(\text { Rich }_{\text {Correct }}+0.5\right) *\left(\text { Lean }_{\text {Incorrect }}+0.5\right)}{\left(\text { Rich }_{\text {Incorrect }}+0.5\right) *\left(\text { Lean }_{\text {Correct }}+0.5\right)}\right)$

High bias values are produced by high numbers of correct responses for rich trials and incorrect responses for lean trials. Discriminability is calculated using the following $\log d$ equation:

$\log d=0.5 * \log \left(\frac{\left(\text { Rich }_{\text {Correct }}+0.5\right) *\left(\text { Lean }_{\text {Correct }}+0.5\right)}{\left(\text { Rich }_{\text {Incorrect }}+0.5\right) *\left(\text { Lean }_{\text {Incorrect }}+0.5\right)}\right)$

High discriminability values are produced by high numbers of correct responses for both rich and lean trials. ( 0.5 is added to all parameters in both equations to avoid instances where no errors are made on a given trial type, thus making log transforms impossible.) The utility of these equations has been repeatedly confirmed in prior studies in humans $[37,42-44,46,60,61]$ and laboratory animals [47-51]. In addition, accuracy (percent correct) and reaction time (latency from line presentation to response) were calculated and presented as individual subject values and session-wide group means ( \pm SEM) for rich and lean trials. Unpaired $t$-tests and Cohen's $d$ were used to evaluate, respectively, group differences and effect sizes (control vs. ELA) within a cohort or with both cohorts combined in $\log b, \log d$, reaction time, sessions to meet discrimination training criteria, body weight, sucrose preference, and entropy. Accuracy during rich and lean trial types during the 5-session PRT testing condition was analyzed using a three-way repeated-measures ANOVA, with "session" and "stimulus" as repeated factors and "experience" as a between subject's factor. Relationships among $\log b$, sucrose preference, and entropy were evaluated via Pearson's correlation coefficient. The criterion for significance was set at $p<0.05$. All statistical analyses were conducted using GraphPad Prism 9 Software (San Diego, CA, USA).

\section{RESULTS}

Reward responsiveness in the PRT is blunted in ELA rats

Figure 2 summarizes group mean ( \pm SEM) and individual subject data across PRT outcomes in control (filled symbols) and ELA subjects (unfilled symbols) from Cohort 1 (left panels), Cohort 2 (middle panels), and both cohorts combined (right panels). Critically, and in line with our hypothesis, significant group differences were observed when examining response biases in favor of the rich stimulus averaged across the 5-session PRT testing condition (upper panels), with subjects in the ELA group exhibiting smaller log $b$ values relative to control subjects in Cohort $1(t[10]=3.50, p=0.006 ; d=2.02)$, Cohort $2(t[18]=3.20, p=0.005 ; d=1.48)$, and when examining both cohorts combined $(t[30]=4.50, p<0.0001 ; d=1.62)$. In contrast to the response bias findings, groups did not differ in their discriminability values $(\log d)$ during the same 5-session PRT testing condition (middle row) in Cohort $1(t[10]=0.13, p=0.90 ; d=0.07)$, Cohort $2(t[18]=0.88, p=0.39 ; d=0.38)$, or when examining both cohorts combined $(t[30]=0.62, p=0.54 ; d=0.22$ ). Likewise, all subjects in both groups completed all 100-trial PRT testing sessions with reaction times from line length stimulus onset to touchscreen response (lower row) that did not differ between groups in Cohort 1 

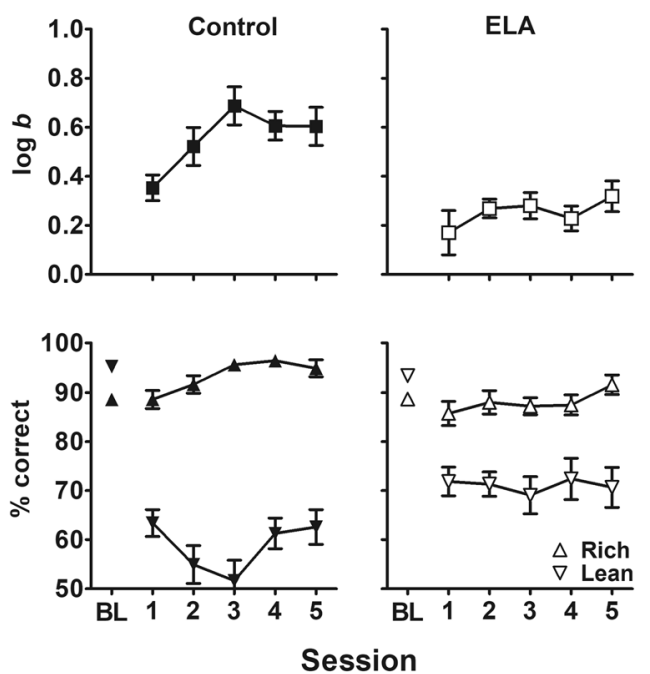

Fig. 3 Development of PRT outcomes by group. Group mean ( \pm SEM) log $b$ (upper panels) and accuracy (lower panels) during rich (triangles) and lean (inverted triangles) stimulus trial types during the last two sessions of baseline (BL) discrimination training and across the five sessions of PRT testing in both cohorts of control (left panels; $n=17$ ) and ELA (right panels; $n=15$ ) subjects.

$(t[10]=1.18, p=0.27 ; d=0.68)$, Cohort $2(t[18]=0.62, p=0.54 ;$ $d=0.28)$, or when examining both cohorts combined $(t[30]=0.01$, $p=0.99 ; d=0.004$ ).

To further interrogate the group differences in response bias, Fig. 3 presents the development of $\log b$ values (top panels) and accuracy by rich/lean trial type (bottom panels) in both cohorts combined across the 5-session PRT testing condition. As the top panels of Fig. 3 show, log $b$ values in the control group were approximately 0.3 in the first test session and increased across subsequent test sessions, reaching an asymptote of approximately 0.6 , whereas $\log b$ values in the ELA group remained stagnant at approximately $0.2-0.3$ throughout the 5 -session testing condition. As the baseline (BL) values plotted in the lower panels of Fig. 3 show, discrimination accuracy was nearly identical between groups immediately prior to exposure to the 3:1 rich:lean probabilistic contingencies. Following exposure to asymmetric probabilistic contingencies, accuracy for the rich trial type was higher than lean accuracy in both groups. However, the disparity between accuracy for rich and lean trial types was larger in the control group. That is, across the 5 -session testing condition, the control group was characterized by consistently higher rich accuracies and lower lean accuracies, relative to the ELA group. Indeed, these early-life experiences produced significant alterations in the development of the expected response bias. For brevity, only effects involving "experience" are reported here. There was no interaction of session $\times$ stimulus $x$ experience $(F[4,120]=1.76, \quad p=0.14)$; however, there was a significant stimulus $\times$ experience interaction $(F[1,30]=19.25, p=0.0001)$. Moreover, the disparity between accuracy for rich $(94.46 \pm 1.14)$ and lean $(58.78 \pm 2.56)$ trial types was larger in the control group compared to that of rich $(88.00 \pm 1.22)$ and lean $(71.07 \pm 2.62)$ in the ELA group. These PRT accuracy outcomes illustrate how log $b$ values in the control group were significantly higher than the ELA group, despite the fact that log $d$ values were similar between groups (cf. Fig. 2). Importantly, as described above, the line length stimulus (long and short) for each subject that was designated as rich and lean was defined by assigning the line length with higher baseline accuracy as lean. Therefore, the response biases observed in both groups during the subsequent 5 -session PRT testing condition were a product of the subject's responsivity to the asymmetric probabilistic contingencies arranged, rather than simply an inflation of an inherent bias.
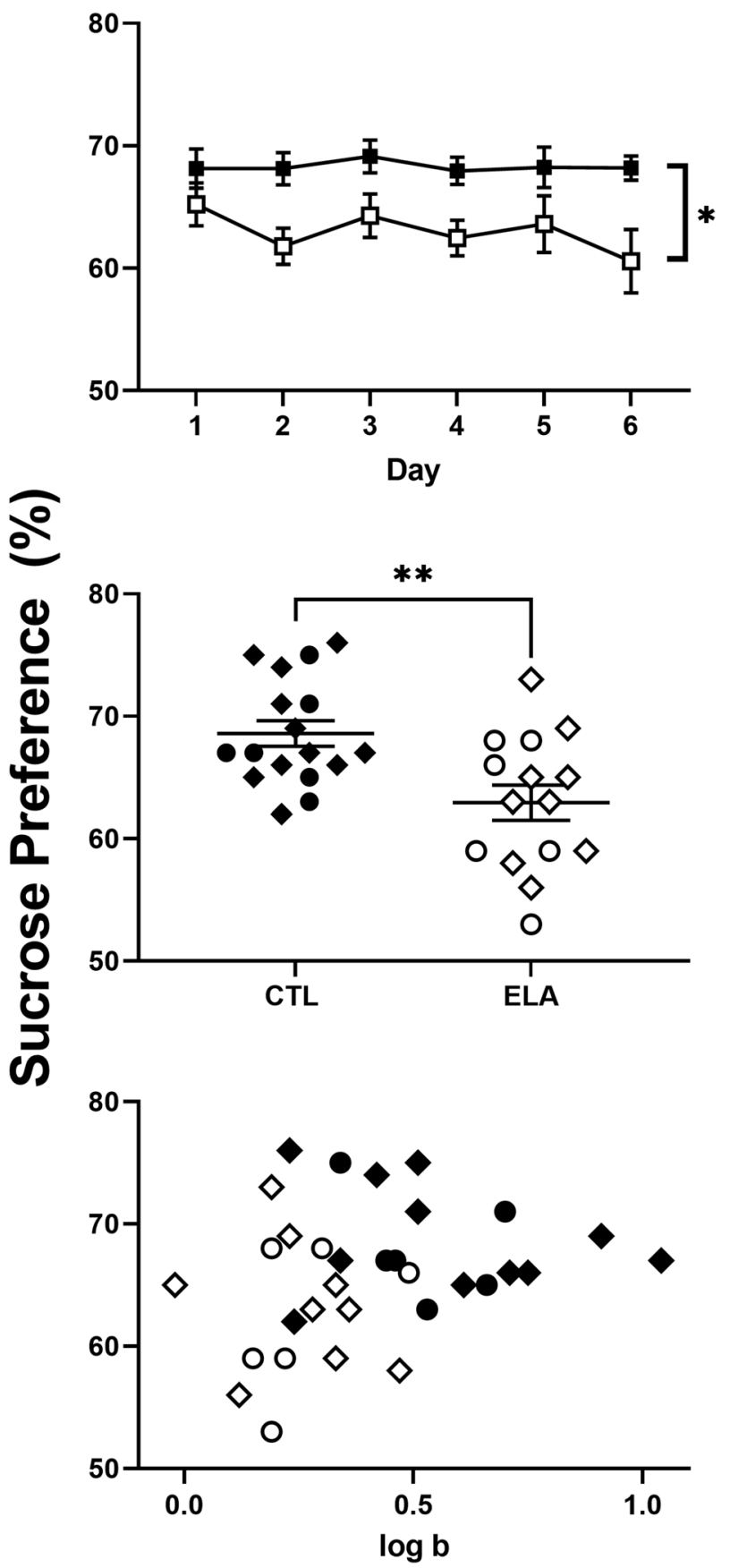

Fig. 4 Sucrose preference outcomes by group and cohort. Top panel: effects of control (filled symbols; $n=17$ ) and ELA (unfilled symbols; $n=15$ ) rearing conditions on mean ( \pm SEM) sucrose preference across the 6-day testing condition. Middle panel: condition-wide mean sucrose preference values in control (filled symbols) and ELA (unfilled symbols) subjects in Cohort 1 (circles) and Cohort 2 (diamonds). Horizontal lines represent group mean $( \pm S E M)$ and data points represent values for individual subjects. Bottom panel: relationship between sucrose preference and log $b$ values in control (filled symbols) and ELA subjects (unfilled symbols) in Cohort 1 (circles) and Cohort 2 (diamonds).

\section{Sucrose preference is blunted in ELA rats}

Preference for sucrose solution was measured in adult animals reared in standard control (filled symbols) or ELA conditions (unfilled symbols). As the top panel of Fig. 4 illustrates, ELA rearing conditions significantly blunted sucrose preference across the 6 -day condition $(F[1,30]=9.74, p=0.004)$. The middle panel of 

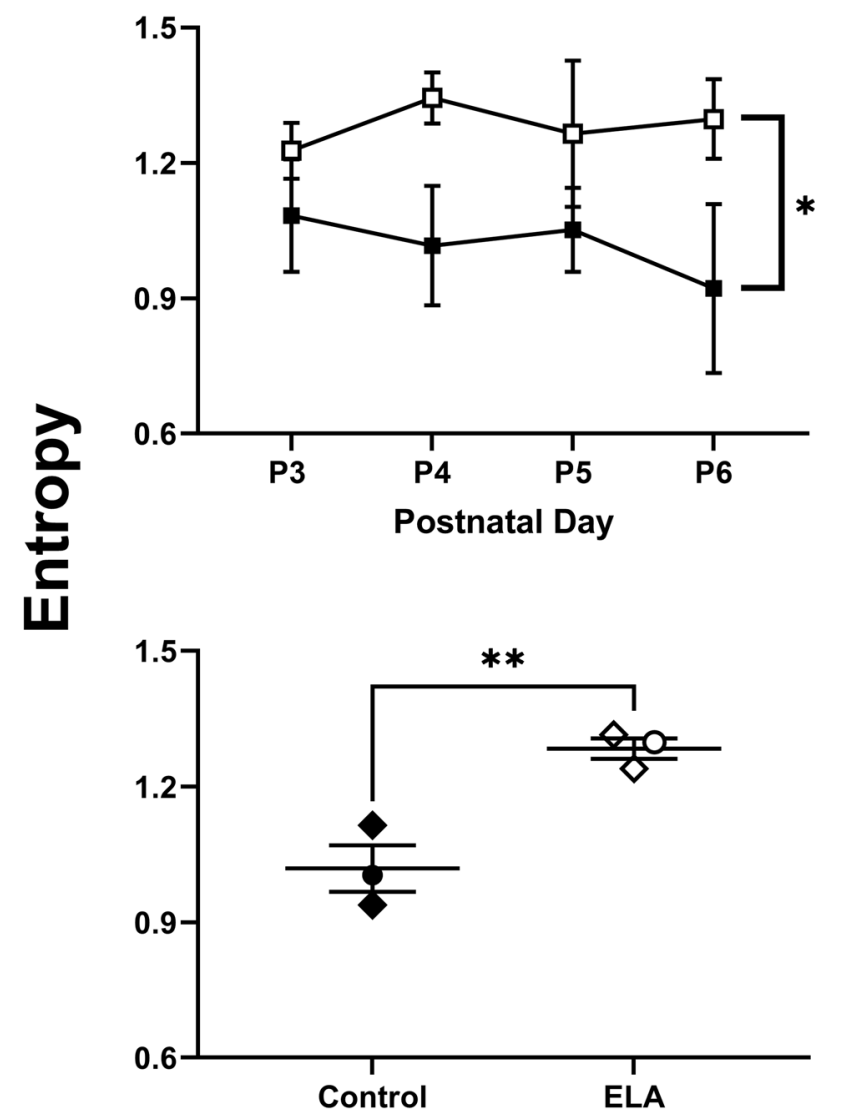

Fig. 5 Maternal unpredictability by group, cohort, and litter. Top panel: effects of control (filled symbols; $n=17$ ) and ELA (unfilled symbols; $n=15$ ) rearing conditions on mean ( \pm SEM) entropy values across P3-P6. Bottom panel: condition-wide mean entropy values in individual control (filled symbols) and ELA (unfilled symbols) dam/ pup interactions in Cohort 1 (circles) and Cohort 2 (diamonds). Horizontal lines represent group mean $( \pm$ SEM), $n=3$ litters per group.

Fig. 4 displays individual subject data in Cohort 1 (circles) and Cohort 2 (diamonds) using the mean preference across the 6 -session condition and reveals significantly lower sucrose preference in ELA subjects relative to control $(t[30]=3.24, p=$ $0.002 ; d=1.14)$. There were no significant differences $(t[30]=0.04$, $p=0.97)$ in mean daily total fluid consumption between control $(61.28 \pm 0.88)$ and ELA $(61.22 \pm 1.28)$ subjects. The bottom panel of Fig. 4 presents the relationship between sucrose preference and $\log b$ in the control subjects (filled symbols) and ELA subjects (unfilled symbols) in Cohort 1 (circles) and Cohort 2 (diamonds). In the control subjects, there was a modest negative correlation between the two measures; however, it did not meet statistical criteria $\left(r=-0.31, R^{2}=0.10, p=0.23\right)$. In the ELA subjects, there was no correlation between sucrose preference and log $b$ $\left(r=-0.12, R^{2}=0.02, p=0.66\right)$.

\section{Probing the salient aspects of ELA that result in reward sensitivity deficits}

ELA in humans and rodents is multifaceted. It is characterized by stress and elevation of stress hormones, but it is unclear whether the activation of the hypothalamic-pituitary adrenal stress axis is directly responsible for enduring behavioral deficits. More recently, evidence has emerged suggesting the maturation of the reward circuit after ELA may be disrupted by aberrant, unpredictable environmental signals during $\operatorname{ELA}[3,8,62]$. Indeed, the need for appropriate signals for circuit development has been shown for both visual and auditory systems [63, 64]. To test whether unpredictable, chaotic signals from the dam to the pups might contribute to the reward-response deficits, we computed an entropy rate, as described previously $[8,57]$. This involves examining a matrix of conditional probabilities that a given maternal behavior " $A$ " is followed by a second behavior " $B$ ". In this context, higher entropy values are indicative of increased unpredictability. As shown in the top panel of Fig. 5, across P3-P6, entropy values were consistently higher in the ELA group relative to control, confirmed by a main effect of ELA $(F[1,4]=$ $22.11, p=0.009)$ with no effect of time $(F[2.52,10.08]=0.1, p=$ $0.94)$ or a time $x$ ELA interaction $(F[3,12]=0.32, p=0.81)$. As there was no significant change in entropy over time, the mean entropy was calculated to create a single value for each litter in Cohort 1 and 2 as shown in the bottom panel of Fig. 5 which also revealed significant differences between control and ELA subjects $(t[4]=$ 4.71, $p=0.009$ ).

\section{Addressing potential confounders}

The studies above employed two cohorts that had modest variations. In addition, it is conceivable that other variables such as body weight might confound the results. Therefore, Table 1 presents subject details by cohort, including the number of pups per dam, date of birth, shipping date from California to Massachusetts, and mean $( \pm$ SEM) body weight (g) P10, P21, $\mathrm{P} 62$, and P78, following clearance from quarantine, and during the 5-session PRT testing condition. Body weights, between Cohort 1 and 2 and between control and ELA subjects, were highly similar throughout developmental stages from rat infancy to adulthood. Most critically, average body weight during the food-restricted 5-session PRT testing conditions was also similar $(t[30]=1.17, p=0.25 ; d=0.41)$ between control $(363.56[ \pm 4.29] \mathrm{g})$ and ELA (372.38 $[ \pm 6.44] \mathrm{g})$ subjects. There were, however, several notable differences between cohorts. First, subjects in Cohort 1 originated from 1 control and 1 ELA dam that each contributed 6 pups to the study, whereas subjects in Cohort 2 originated from 2 control dams contributing 6 and 5 pups and 2 ELA dams contributing 5 and 4 pups. Second, Cohort 1 was 17 days older than Cohort 2 when they were shipped from California to Massachusetts. Third, despite being younger at ship date, because of delays related to COVID-19 in sentinel testing, Cohort 2 spent approximately 30 more days in quarantine than Cohort 1 that made them slightly older during PRT training and testing. Despite these differences, all subjects learned to touch the screen and discriminate long vs. short lines. The control and ELA groups met discrimination criteria of $\geq 80 \%$ correct during both long and short line length trial types for two consecutive sessions following, respectively, $12.24( \pm 0.76)$ and $14.80( \pm 1.65)$ training sessions, a group difference that did not meet statistical criteria $(t[30]=1.47$, $p=0.15 ; d=0.51)$.

\section{DISCUSSION}

The present studies identify the PRT as a powerful translational tool that can advance understanding of mechanisms underlying reward responsiveness across species. Using this assay, we identified enduring effects of ELA on reward responsiveness during adulthood. Specifically, blunted development of a response bias in the PRT was observed, analogous to previous studies in human participants with anhedonia. Anhedonia-related phenotypes were also observed in these rats when tested in the traditional sucrose preference test. The level of unpredictability in maternal care was significantly higher in subjects that would later display blunted reward responsiveness in both the PRT and the sucrose preference test during adulthood, highlighting predictability early in life as a salient signal for reward system development.

Maternal patterns and signals are an important factor in the regulation of brain circuits $[63,64]$. Recent work in humans has verified that unpredictable maternally derived signals early in 
development are correlated with worse cognitive outcomes later in life $[6,65]$. In rodents, chronic unpredictable and fragmented maternal care causes disruption to the sensory signals received by the pups (without causing physical stress [66] and minimal effects on other aspects of maternal care [8,53, 67-69], resulting in enduring cognitive and emotional deficits $[8,55,68])$. Evidence from the literature also suggests that predictable maternal care is required for reward responsiveness. Previous studies using maternal separation as an early-life stressor have demonstrated anhedonia-related phenotypes in adulthood are observed only after a secondary stressor is experienced [70, 71]. However, unpredictability in maternal care has been shown to directly induce anhedonia-related behavior in adulthood as measured by sucrose preference and social play $[3,8]$. Exposure to a secondary stressor can accelerate the effects of unpredictable maternal care on learning and memory [72]. Therefore, it is conceivable that rats experiencing ELA are more vulnerable to stress, such as those caused by social manipulations during testing and in transit. The present studies add to this research domain by identifying the direct correlations between the magnitude of maternal entropy and reward responsiveness later in life.

Interestingly, although group differences were observed in both assays, their respective primary metrics (i.e., preference for the sucrose bottle and $\log b$ ) did not correlate with each other among individual subjects. Reasons for this are currently unclear; while this study is only powered to identify large effect sizes, anhedonia is a construct with high heterogeneity and neither sucrose preference nor the PRT captures its full spectrum. In fact, they likely evaluate different aspects of reward responsiveness (reward consumption vs. reward learning). Therefore, the lack of correlation between assay outcomes is perhaps not surprising but, rather, consistent with an RDoC-like framework that requires a diversity of tasks to investigate multidimensional psychological systems $[35,36]$. These novel findings, which utilize a reversetranslated assay used with clinical patients to quantify deficits in reward responsiveness, are also highly consistent with longstanding literature on childhood traumas associated with subsequent vulnerability to anhedonia that can persist throughout life [18-22]. They are similarly concordant with rodent studies describing altered reward system function and development following ELA $[3,8,62]$.

There are a few caveats that warrant consideration. First, although significant group differences were observed across two cohorts of subjects, both sucrose preference and PRT outcomes were each examined at only one timepoint-early and middle adulthood, respectively. Although it would be interesting to detail the time course of how quickly this blunting in reward responsiveness emerges and whether it persists until death, it is nevertheless remarkable that its effects are sustained well into rat adulthood with a magnitude that is clearly distinguishable from control subjects. Moreover, the systematic replication of these findings across 2 years, two cohorts, 32 rats, and 6 litters under somewhat varying conditions supports the robust and reproducible nature of these ELA effects. Second, it is important to note that these studies were conducted on male subjects only. Given the fact that diagnoses of mood disorders in which anhedonia is prominent are more prevalent in women [73] and the known sexdependent effects of ELA on reward [7], it will be important to determine the extent to which these effects are also observed in female rats.

In conclusion, the PRT is a valuable translational assay for elucidating the mechanisms by which ELA impacts the reward circuit in both humans and laboratory animals. The present data confirm a relationship between maternal entropy during early-life and reward responsiveness in adulthood. Moreover, these findings corroborate previous work implicating maternal signals during early-life as modulators of reward circuit signaling specifically and cognitive development generally.

\section{REFERENCES}

1. Authement ME, Kodangattil JN, Gouty S, Rusnak M, Symes AJ, Cox BM, et al. Histone deacetylase inhibition rescues maternal deprivation-induced GABAergic metaplasticity through restoration of AKAP signaling. Neuron. 2015;86:1240-52.

2. Bolton JL, Ruiz CM, Rismanchi N, Sanchez GA, Castillo E, Huang J, et al. Early-life adversity facilitates acquisition of cocaine self-administration and induces persistent anhedonia. Neurobiol Stress. 2018;8:57-67.

3. Bolton JL, Molet J, Regev L, Chen Y, Rismanchi N, Haddad E, et al. Anhedonia following early-life adversity involves aberrant interaction of reward and anxiety circuits and is reversed by partial silencing of amygdala corticotropin-releasing hormone gene. Biol Psychiatry. 2018;83:137-47.

4. Brodnik ZD, Black EM, España RA. Accelerated development of cocaineassociated dopamine transients and cocaine use vulnerability following traumatic stress. Neuropsychopharmacology. 2019;45:472-81.

5. Chen Y, Baram TZ. Toward understanding how early-life stress reprograms cognitive and emotional brain networks. Neuropsychopharmacology. 2016;41:197-206.

6. Davis EP, Stout SA, Molet J, Vegetabile B, Glynn LM, Sandman CA, et al. Exposure to unpredictable maternal sensory signals influences cognitive development across species. Proc Natl Acad Sci USA. 2017;114:10390-5.

7. Levis SC, Bentzley BS, Molet J, Bolton JL, Perrone CR, Baram TZ, et al. On the early life origins of vulnerability to opioid addiction. Mol Psychiatry. 2021;26:4409-16.

8. Molet J, Heins K, Zhuo X, Mei YT, Regev L, Baram TZ, et al. Fragmentation and high entropy of neonatal experience predict adolescent emotional outcome. Transl Psychiatry. 2016;6:e702.

9. Shepard RD, Nugent FS. Early life stress- and drug-induced histone modifications within the ventral tegmental area. Front Cell Dev Biol. 2020;8:588476.

10. Ulrich-Lai YM, Fulton S, Wilson M, Petrovich G, Rinaman L. Stress exposure, food intake and emotional state. Stress. 2015;18:381-99.

11. Victoria NC, Inoue K, Young LJ, Murphy AZ. Long-term dysregulation of brain corticotrophin and glucocorticoid receptors and stress reactivity by single earlylife pain experience in male and female rats. Psychoneuroendocrinology. 2013;38:3015-28.

12. Bonuck K, McGrath K, Gao Q. National Parent Survey 2017: worries, hopes, and child well-being. J Community Psychol. 2020;48:2532-51.

13. Danese A, Lewis SJ. Psychoneuroimmunology of early-life stress: the hidden wounds of childhood trauma? Neuropsychopharmacology. 2017;42:99-114.

14. Green JG, McLaughlin KA, Berglund PA, Gruber MJ, Sampson NA, Zaslavsky AM, et al. Childhood adversities and adult psychiatric disorders in the national comorbidity survey replication I: associations with first onset of DSM-IV disorders. Arch Gen Psychiatry. 2010;67:113-23.

15. Short AK, Baram TZ. Early-life adversity and neurological disease: age-old questions and novel answers. Nat Rev Neurol. 2019;15:657-69.

16. Silvers JA, Goff B, Gabard-Durnam L, Gee DG, Fareri DS, Caldera C, et al. Vigilance, the amygdala, and anxiety in youths with a history of institutional care. Biol Psychiatry Cogn Neurosci Neuroimaging. 2017;2:493-501.

17. Birnie MT, Kooiker CL, Short AK, Bolton JL, Chen Y, Baram TZ. Plasticity of the reward circuitry after early-life adversity: mechanisms and significance. Biol Psychiatry. 2020;87:875-84.

18. Boecker R, Holz NE, Buchmann AF, Blomeyer D, Plichta MM, Wolf I, et al. Impact of early life adversity on reward processing in young adults: EEG-fMRI results from a prospective study over 25 years. PLoS One. 2014;9:e104185.

19. Dillon DG, Holmes AJ, Birk JL, Brooks N, Lyons-Ruth K, Pizzagalli DA. Childhood adversity is associated with left basal ganglia dysfunction during reward anticipation in adulthood. Biol Psychiatry. 2009;66:206-13.

20. Hanson JL, Hariri AR, Williamson DE. Blunted ventral striatum development in adolescence reflects emotional neglect and predicts depressive symptoms. Biol Psychiatry. 2015;78:598-605.

21. Mehta MA, Gore-Langton E, Golembo N, Colvert E, Williams SCR, Sonuga-Barke E. Hyporesponsive reward anticipation in the basal ganglia following severe institutional deprivation early in life. J Cogn Neurosci. 2010;22:2316-25.

22. Pechtel P, Pizzagalli DA. Effects of early life stress on cognitive and affective function: an integrated review of human literature. Psychopharmacology. 2011;214:55-70.

23. Ventura AK, Worobey J. Early influences on the development of food preferences. Curr Biol. 2013;23:R401-R408.

24. Whitton AE, Treadway MT, Pizzagalli DA. Reward processing dysfunction in major depression, bipolar disorder and schizophrenia. Curr Opin Psychiatry. 2015;28:7-12.

25. Hasler G, Drevets WC, Gould TD, Gottesman II, Manji HK. Toward constructing an endophenotype strategy for bipolar disorders. Biol Psychiatry. 2006;60:93-105.

26. Horan WP, Kring AM, Blanchard JJ. Anhedonia in schizophrenia: a review of assessment strategies. Schizophr Bull. 2006;32:259-73.

27. Nawijn L, van Zuiden M, Frijling JL, Koch SBJ, Veltman DJ, Olff M. Reward functioning in PTSD: a systematic review exploring the mechanisms underlying anhedonia. Neurosci Biobehav Rev. 2015;51:189-204. 
28. Risbrough VB, Glynn LM, Davis EP, Sandman CA, Obenaus A, Stern HS, et al. Does anhedonia presage increased risk of posttraumatic stress disorder? In: Vermetten E, Baker D, Risbrough V, editors. Behavioral Neurobiology of PTSD. Vol. 38. Cham: Springer International Publishing; 2018. p. 249-65.

29. Markou A, Kosten TR, Koob GF. Neurobiological similarities in depression and drug dependence: a self-medication hypothesis. Neuropsychopharmacology. 1998;18:135-74.

30. Rizvi SJ, Pizzagalli DA, Sproule BA, Kennedy SH. Assessing anhedonia in depression: potentials and pitfalls. Neurosci Biobehav Rev. 2016;65:21-35.

31. Snaith RP, Hamilton M, Morley S, Humayan A, Hargreaves D, Trigwell P. A scale for the assessment of hedonic tone the Snaith-Hamilton Pleasure Scale. Br J Psychiatry. 1995;167:99-103.

32. Chapman L, Chapman JP, Raulin ML. Scales for physical and social anhedonia. J Abnorm Psychol. 1976;85:374-82.

33. Fawcett J. Assessing anhedonia in psychiatric patients. Arch Gen Psychiatry. 1983;40:79.

34. Scheggi S, De Montis MG, Gambarana C. Making sense of rodent models of anhedonia. Int J Neuropsychopharmacol. 2018;21:1049-65.

35. National Institute of Mental Health. Behavioral assessment methods for RDoC constructs (revised August 2016). 2016. https://www.nimh.nih.gov/about/ advisory-boards-and-groups/namhc/reports/behavioral-assessment-methodsfor-rdoc-constructs. Accessed 1 Sep 2021

36. Insel T, Cuthbert B, Garvey M, Heinssen R, Pine DS, Quinn K, et al. Research Domain Criteria (RDoC): toward a new classification framework for research on mental disorders. Am J Psychiatry. 2010;167:748-51.

37. Pizzagalli DA, Jahn AL, O'Shea JP. Toward an objective characterization of an anhedonic phenotype: a signal-detection approach. Biol Psychiatry. 2005;57:319-27.

38. Tripp G, Alsop B. Sensitivity to reward frequency in boys with attention deficit hyperactivity disorder. J Clin Child Psychol. 1999;28:366-75.

39. Luc OT, Pizzagalli DA, Kangas BD. Toward a quantification of anhedonia: unified matching law and signal detection for clinical assessment and drug development. Perspect Behav Sci. 2021. https://doi.org/10.1007/s40614-021-00288-w.

40. Macmillan NA, Creelman CD. Detection theory: a user's guide. New York, NY, US: Cambridge University Press; 1991.

41. McCarthy D, Davison M. Signal probability, reinforcement and signal detection. J Exp Anal Behav. 1979;32:373-86.

42. Fletcher K, Parker G, Paterson A, Fava M, losifescu D, Pizzagalli DA. Anhedonia in melancholic and non-melancholic depressive disorders. J Affect Disord. 2015; $184: 81-88$

43. Pizzagalli DA, Goetz E, Ostacher M, losifescu DV, Perlis RH. Euthymic patients with bipolar disorder show decreased reward learning in a probabilistic reward task. Biol Psychiatry. 2008;64:162-8.

44. Pizzagalli DA, losifescu D, Hallett LA, Ratner KG, Fava M. Reduced hedonic capacity in major depressive disorder: evidence from a probabilistic reward task. J Psychiatr Res. 2008;43:76-87.

45. Whitton AE, Green Al, Pizzagalli DA, Roth RM, Williams JM, Brunette MF. Potent dopamine D2 antagonists block the reward-enhancing effects of nicotine in smokers with schizophrenia. Schizophr Bull. 2019;45:1300-8.

46. Vrieze E, Pizzagalli DA, Demyttenaere $K$, Hompes $T$, Sienaert $P$, De Boer $P$, et al. Reduced reward learning predicts outcome in major depressive disorder. Biol Psychiatry. 2013;73:639-45.

47. Der-Avakian A, D'Souza MS, Pizzagalli DA, Markou A. Assessment of reward responsiveness in the response bias probabilistic reward task in rats: implications for cross-species translational research. Transl Psychiatry. 2013;3:e297.

48. Kangas BD, Wooldridge LM, Luc OT, Bergman J, Pizzagalli DA. Empirical validation of a touchscreen probabilistic reward task in rats. Transl Psychiatry. 2020;10:285.

49. Wooldridge LM, Bergman J, Pizzagalli DA, Kangas BD. Translational assessments of reward responsiveness in the marmoset. Int J Neuropsychopharmacol. 2021;24:409-18.

50. Lamontagne SJ, Melendez Sl, Olmstead MC. Investigating dopamine and glucocorticoid systems as underlying mechanisms of anhedonia. Psychopharmacology. 2018;235:3103-13.

51. Der-Avakian A, D'Souza MS, Potter DN, Chartoff EH, Carlezon WA, Pizzagalli DA, et al. Social defeat disrupts reward learning and potentiates striatal nociceptin/ orphanin FQ mRNA in rats. Psychopharmacology. 2017;234:1603-14.

52. National Research Council. Guide for the care and use of laboratory animals. 8th ed. Washington, DC: National Academies Press; 2011.

53. Gilles EE, Schultz L, Baram TZ. Abnormal corticosterone regulation in an immature rat model of continuous chronic stress. Pediatr Neurol. 1996;15:114-9.

54. Molet J, Maras PM, Avishai-Eliner S, Baram TZ. Naturalistic rodent models of chronic early-life stress. Dev Psychobiol. 2014;56:1675-88.

55. Walker C-DC, Bath KKG, Joels M, Korosi A, Larauche M, Lucassen PJ, et al. Chronic early life stress induced by limited bedding and nesting (LBN) material in rodents: critical considerations of methodology, outcomes and translational potential. Stress. 2017;20:421-48
56. Friard O, Gamba M. BORIS: a free, versatile open-source event-logging software for video/audio coding and live observations. Methods Ecol Evol. 2016;7:1325-30.

57. Vegetabile BG, Stout-Oswald SA, Davis EP, Baram TZ, Stern HS. Estimating the entropy rate of finite markov chains with application to behavior studies. J Educ Behav Stat. 2019;44:282-308.

58. Kangas BD, Bergman J. Touchscreen technology in the study of cognition-related behavior. Behav Pharm. 2017;28:623-9.

59. Kangas BD, Branch MN. Empirical validation of a procedure to correct position and stimulus biases in matching-to-sample. J Exp Anal Behav. 2008;90:103-12.

60. Boger KD, Auerbach RP, Pechtel P, Busch AB, Greenfield SF, Pizzagalli DA. Co-occurring depressive and substance use disorders in adolescents: an examination of reward responsiveness during treatment. J Psychother Integr. 2014;24:109-21.

61. Janes $A C$, Pedrelli $P$, Whitton $A E$, Pechtel $P$, Douglas $S$, Martinson $M A$, et al. Reward responsiveness varies by smoking status in women with a history of major depressive disorder. Neuropsychopharmacology. 2015;40:1940-6.

62. Wendel KM, Short AK, Noarbe BP, Haddad E, Palma AM, Yassa MA, et al. Early life adversity in male mice sculpts reward circuits. Neurobiol Stress. 2021;15:100409.

63. Li Y, Fitzpatrick D, White LE. The development of direction selectivity in ferret visual cortex requires early visual experience. Nat Neurosci. 2006;9:676-81.

64. Zhang $\mathrm{LI}$, Bao S, Merzenich MM. Persistent and specific influences of early acoustic environments on primary auditory cortex. Nat Neurosci. 2001;4:1123-30.

65. Davis EP, Korja R, Karlsson L, Glynn LM, Sandman CA, Vegetabile B, et al. Across continents and demographics, unpredictable maternal signals are associated with children's cognitive function. EBioMedicine. 2019;46:256-63.

66. Bolton JL, Short AK, Simeone KA, Daglian J, Baram TZ. Programming of stresssensitive neurons and circuits by early-life experiences. Front Behav Neurosci. 2019;13:30.

67. Brunson KL, Kramár E, Lin B, Chen $\mathrm{Y}$, Colgin LL, Yanagihara TK, et al. Mechanisms of late-onset cognitive decline after early-life stress. J Neurosci. 2005;25:9328-38.

68. Ivy AS, Brunson KL, Sandman C, Baram TZ. Dysfunctional nurturing behavior in rat dams with limited access to nesting material: a clinically relevant model for earlylife stress. Neuroscience. 2008;154:1132-42.

69. Rice CJ, Sandman CA, Lenjavi MR, Baram TZ. A novel mouse model for acute and long-lasting consequences of early life stress. Endocrinology. 2008;149:4892-4900.

70. Hill RA, Klug M, Kiss Von Soly S, Binder MD, Hannan AJ, van den Buuse M. Sexspecific disruptions in spatial memory and anhedonia in a "two hit" rat model correspond with alterations in hippocampal brain-derived neurotrophic factor expression and signaling. Hippocampus. 2014;24:1197-211.

71. Uchida S, Hara K, Kobayashi A, Funato H, Hobara T, Otsuki K, et al. Early life stress enhances behavioral vulnerability to stress through the activation of REST4mediated gene transcription in the medial prefrontal cortex of rodents. J Neurosci. 2010;30:15007-18.

72. Molet J, Maras PM, Kinney-Lang E, Harris NG, Rashid F, Ivy AS, et al. MRI uncovers disrupted hippocampal microstructure that underlies memory impairments after early-life adversity. Hippocampus. 2016;26:1618-32.

73. Hyde JS, Mezulis AH. Gender differences in depression: biological, affective, cognitive, and sociocultural factors. Harv Rev Psychiatry. 2020;28:4-13.

\section{AUTHOR CONTRIBUTIONS}

$B D K, A K S, T Z B$, and DAP designed the research, AKS and OTL performed the research, $\mathrm{BDK}, \mathrm{AKS}, \mathrm{OTL}$, and HSS analyzed the data, and BDK, AKS, TZB, and DAP wrote the paper.

\section{FUNDING}

BDK was partially supported by R01-DA047575 from the National Institute on Drug Abuse. AKS and TZB were partially supported by P50-MH96689 and R01-MH73136 from the National Insitiute of Mental Health. DAP was partially supported by R01MH101521, R37-MH068376, and P50-MH119467 from the National Institute of Mental Health. The content is solely the responsibility of the authors and does not necessarily represent the official views of the National Institutes of Health.

\section{COMPETING INTERESTS}

Over the past 3 years, DAP has received funding from $\mathrm{NIMH}$, Brain and Behavior Research Foundation, the Dana Foundation, and Millennium Pharmaceuticals; 
consulting fees from Albright Stonebridge Group, BlackThorn Therapeutics, Boehringer Ingelheim, Compass Pathway, Concert Pharmaceuticals, Engrail Therapeutics, Neurocrine Biosciences, Otsuka Pharmaceuticals, and Takeda Pharmaceuticals; one honorarium from Alkermes; stock options from BlackThorn Therapeutics. DAP has a financial interest in BlackThorn Therapeutics, which has licensed the copyright to the Probabilistic Reward Task through Harvard University. DAP's interests were reviewed and are managed by McLean Hospital and Partners HealthCare in accordance with their conflict of interest policies. All other authors report no biomedical financial interests or potential conflicts of interest.

\section{ADDITIONAL INFORMATION}

Correspondence and requests for materials should be addressed to Diego A. Pizzagalli.

Reprints and permission information is available at http://www.nature.com/ reprints

Publisher's note Springer Nature remains neutral with regard to jurisdictional claims in published maps and institutional affiliations. 